\title{
The role of JNK 1 in controlling AQP-1 and choroidal thickness during recovery from form-deprivation myopia in guinea pigs
}

\section{Wei Chen}

Tianjin Eye Hospital

\section{Li zhi Wei}

Shandong Provincial Hospital

\section{Wang qi Miao}

Tianjin Eye Hospital

\section{Wang Yan}

tianjin eye hospital

Zhang Yue ( $\nabla$ zmoon1976@sina.com )

Research article

Keywords: p-JNK1, AQP-1, choroidal thickness, recovery, form deprivation

Posted Date: June 8th, 2020

DOl: https://doi.org/10.21203/rs.3.rs-19152/v2

License: (c) (1) This work is licensed under a Creative Commons Attribution 4.0 International License. Read Full License 


\section{Abstract}

Background: The development and recovery(REC) of myopia is associated with changing of choroidal thickness(CT) in model of guinea pigs. In this process, Aquaporin-1 (AQP-1) is related to changes in choroidal thickness during the recovery from myopia, but the corresponding signaling pathway has not been clarified. The aim of this study was to investigate the effect of JNK1 on CT/AQP-1 and the recovery from myopia.

Methods: According to the different intravitreal injections in eyes that underwent form deprivation for 21 days, guinea pigs were divided into four groups: the REC group, the REC+anisomycin (agonist for JNK1, REC-AN) group, the REC+SP600125 (inhibitor for JNK1, REC-SP) group, and the REC+DMSO (REC-DM) group. Each group was divided into three subgroups according to the time of removal of the deprivation: 3 days (d), $7 \mathrm{~d}$ and $10 \mathrm{~d}$. All animals underwent biometric measurements (refractive error, axial length $(\mathrm{AL})$, and $\mathrm{CT}$ ), and the protein expression of AQP-1 and p-JNK1 in the choroid was also measured.

Results: In the REC and REC-DM groups, significant differences in CT/ refractive error /AL/p-JNK1 or AQP1 were only found in the $3 d$ group compared with the normal control $(N C)$ group (all $p<0.05$ ). In the RECAN group, CT/p-JNK1 or AQP-1 in the $3 d$ group was significantly higher than that in the other $3 d^{\prime}$ groups (all $p<0.05$ ), but no significant difference in refractive error or $A L$ was found compared with that in the NC group (all p>0.05). In the REC-SP group, a significant difference in refractive error /CT/p-JNK1 or AQP1 was found in the $3 d / 7 d$ group compared with the NC group (all $p<0.05$ ), but AL was only found in the $3 d$ groups (both $p=0.001)$.

Conclusions: Changes in JNK1 phosphorylation can regulate AQP-1 and CT during the recovery from myopia and the recovery time. Thus, JNK1 may be a potential therapeutic target for preventing/treating myopia.

\section{Background}

Myopia is the most common visual disease worldwide. The prevalence of myopia in children in developed countries of East and Southeast Asia is estimated to be $80-90 \%$ [1]. Although our knowledge of the mechanisms that underlie the development and progression of myopia remains limited, studies in guinea pig models of myopia have shown that recovery from myopia can occur after the deprived eye is re-exposed to a normal environment [2]. In guinea pigs, the choroid can decrease its thickness in response to form deprivation-induced myopia, while it can increase its thickness after the deprived eye is reexposed to unrestricted vision measured by optical coherence tomography (OCT) [3]. This finding suggested that the choroid might participate in refractive adjustment as an accommodative mechanism, but the mechanism of choroidal regulation is still unclear.

The choroid is a dense network of blood vessels with multiple functions. Specifically, the choroid provides nourishment to the outer layers of the retina and participates in refractive adjustment. In addition, choroidal secretory cells secrete growth factors that modulate vascularization and growth of the sclera or 
affect the molecular signals to the sclera [4]. The causes of changes in choroidal thickness(CT) include choroidal blood flow [5], lymphatics [6], nonvascular smooth muscle [7], osmotic macromolecules [8] and suprachoroidal volume [3]. Aquaporin-1 (AQP-1) is a water channel protein that selectively allows the passage of water through the plasma membrane of fluid-transporting cells throughout the body [9]. Chen et al. [3] found that AQP-1 is highly expressed in choroidal endothelial cells and has a positive correlation with $\mathrm{CT}$ and the recovery from form-deprivation myopia in guinea pigs, but the mechanism of optical signaling regulating AQP-1 and choroidal thickness has not been studied.

C-Jun N-terminal kinase-1 (JNK1) is an important member of the mitogen-activated protein kinase family. The JNK signaling pathway can be activated by growth factors, cytokines, environmental stress (UV, cell hyperosmotic pressure, ischemia-reperfusion injury) and other stimulatory signals to regulate the transcription of downstream genes [10]. Brand C et al. found that the mRNA level of mapk8ip3 (a factor that can cause JNK signal pathway activation) decreased during the development of myopia, and the levels of downstream genes of JNK, such as c-fos, were also reduced [11]. In a previous study on AQP1 activation, Umenishi $F$ et al. found that the expression of AQP-1 increased after activation of JNK in rat bone marrow cells and decreased after inhibition of JNK [12], indicating that the JNK pathway can affect AQP1 activation. Whether JNK1 regulates AQP-1 during the recovery from myopia has not been studied. We speculate that changes in the JNK1 signaling pathway may lead to changes in AQP-1 content and CT, thus influencing the recovery from myopia.

The purpose of this study was to observe the changes in choroidal AQP1 and refractive error in guinea pigs during recovery from myopia by regulating the content of JNK1, and changes in CT were also observed.

\section{Methods}

Animals

A total of 136 pigmented guinea pigs (3 weeks old) from the Beijing Keyu Animal Centre (Beijing, China) were raised on a daily cycle of 12-h illumination (500 Lux) and 12-h darkness during the experimental period (on at 8:00 AM, off at 8:00 PM), with free access to water and food supplemented with vitamin C. Form-deprivation myopia was induced by the animals wearing a latex face mask (Suzhou, China) on the right eye. The animals were monitored 3 times during the 12-hour light period to ensure that the face masks were in place and were promptly changed to a suitable size when necessary. These face masks were made by latices, which were opaque and soft with light transmission of $60 \%$. The face masks did not contact the cornea, which allowed the right eyes to blink freely. The animal research in this study was approved by the Animal Care and Ethics Committee at Tian Jin Eye Hospital, Tianjin, China. The treatment and care of the animals complied with the ARVO Statement for the Use of Animals in Ophthalmic and Vision Research.

Experimental Design and Groups 
The animals wore the face mask for 21 days to induce myopia. Then, the face masks were removed, and the guinea pigs were re-exposed to the normal visual environment. Ninety-six guinea pigs were divided into four groups: the recovery group (REC, $n=24$ ), the REC plus SP600125 group (REC-SP, $n=24$ ), the REC plus anisomycin group (REC-AN, $n=24$ ) and the REC plus DMSO group (vehicle) (REC-DM, $n=24$ ). SP600125 (Abcam, Cambridge, MA, USA) and anisomycin (Abcam, Cambridge, MA, USA) were injected into the REC-SP group and the REC-AN group, respectively, at the same concentration and volume $(0.2$ $\mathrm{nmol}, 0.01 \mathrm{ml}$ ). SP600125, which is an inhibitor of JNK, and anisomycin, which is an agonist of JNK, were both dissolved in DMSO and diluted with PBS to their final concentrations. In the REC-DM group, the form-deprived eyes were injected with sterilized DMSO solution $(0.01 \mathrm{ml}$, diluted with PBS), which is the carrier. All intravitreal injections were performed once using a microinjector (Shanghai Meter Glass Factory, Shanghai, China) with a 30-gauge needle at $1.5 \mathrm{~mm}$ posterior to the temporal limbus under anesthesia with $2.5 \mathrm{~g} / \mathrm{kg}$ chloral hydrate (intraperitoneal injection) when the face masks were removed. In the REC group, the guinea pigs were raised without any injection after the face mask was removed. Moreover, the normal control group (NC) included 32 guinea pigs that were free of form deprivation and were left untreated.

The animals in the four experimental groups were divided into the following three subgroups $(n=8$, each), based on the length of exposure to the normal environment: 1 ) the $3 d$ group ( 3 days of exposure) the $7 d$ group ( 7 days of exposure), and 3 ) the $10 \mathrm{~d}$ group (10 days of exposure). The normal control group was also divided into three subgroups $(n=8$, each) corresponding to the time of the experimental group. We also added two groups in this experiment: first, the form deprivation for 21 days (FD 21d) group $(n=8)$; second, a normal control for 21 days (NC 21d) group ( $n=8)$ corresponding to the time of the FD 21d group. The randomly assigned experimental animal groups are shown in Table 1.

Refractive Error and Axial Length (AL) Measurements

All animals underwent biometric measurements (refractive error and $A L$ ) at the end of each FD and recovery period on the experimental days. Biometric measurement was performed by a research optometrist who was masked to the treatment group assignment with the help of an animal care assistant.

Refractive errors were measured by hand-held streak retinoscopy on awake, behaving animals. The measurement was performed one hour after instillation of 1 or 2 drops of tropicamide phenylephrine eye drops (Santen Pharmaceutical Co., Ltd., Osaka, Japan) to achieve cycloplegia in a dark room on the experimental days. All refractive error data presented refer to the spherical equivalent (averages of results for the two principal meridians) refraction. Three replicate measures of the same eye were recorded with the mean used for statistical analysis.

Ocular axial length was measured by high frequency A-scan ultrasonography (10-MHz, Suoer, Tianjin, China) on the same day following refraction measurements. Topical cornea anesthesia was carried out by administering a drop of $0.5 \%$ proparacaine hydrochloride (Alcon, Puurs, Belgium) 2 minutes prior to 
the ultrasound measurement on awake animal. The ultrasound probe had direct contact with the cornea during the measurement. The average of 8 measurements was adopted.

\section{Measurement of choroidal thickness}

With the spectral-domain RTVue OCT (Optovue XR-100-2, Fremont, CA, USA), the scan patterns were the retina cross line mode, which consists of 2 orthogonally oriented 9-mm lines consisting of 1024 A-scans after the animals were anaesthetized with $2.5 \mathrm{~g} / \mathrm{kg}$ chloral hydrate (intraperitoneal injection). CT was defined as the distance from the outermost layer of the retinal pigment epithelium (RPE) to the inner scleral border, presumed to be the chorioscleral interface [13], using the caliper system provided by the software. The choroidal thicknesses at $3 \mathrm{~mm}$ from the optic disc of 18 radial lines were measured manually by two independent researchers. The mean overall CT was obtained by calculating the average values of the CT measurements at all eccentricities. If the line was blurred, especially for the chorioscleral interface and RPE interface, the center of the line was traced and measured. Measurements by the first examiner were performed three times to evaluate the intraobserver variability. All measurements were performed at the same time of the day (between 5:00 pm and 9:00 pm) in all guinea pigs to avoid the effect of diurnal CT variation on the results [14]

\section{Collection of Choroid Samples}

Tissue Isolation

At the end of form deprivation and each recovery period, the guinea pigs were terminally euthanized with $2.5 \mathrm{~g} / \mathrm{kg}$ chloral hydrate (intraperitoneal injection), and the right eyes in all animals were enucleated. Using a surgical microscope (Zeiss, American) and razor blade, we cut the eyes of the guinea pigs perpendicular to the anteroposterior axis and approximately $1 \mathrm{~mm}$ posterior to the ora serrata on the ice plate. The anterior segment of the eye was discarded. For isolation of the choroid, the retina and RPE were removed as a sheet with a small spatula, and then, the choroid was carefully removed from the underlying sclera. Occasionally, small bits of RPE adhered to the choroid, and these pieces were removed by gentle brushing with a brush and rinsing with PBS. The tissues were stored in liquid nitrogen at $-80^{\circ} \mathrm{C}$ until use.

\section{Western Blot Analysis}

Total protein was extracted from the frozen choroids of the eye with ice-cold extraction buffer as well as protease inhibitors. Protein was separated on a $10 \%$ resolving gel and transferred to cellulose acetate membrane (Millipore). Then, these membranes were blocked with $5 \%$ nonfat dry milk in $0.1 \%$ Tween 20 for 2 hours at room temperature. Afterwards, these membranes were cultured overnight with a primary antibody against p-JNK 1 (Abcam, Cambridge, MA, USA) and AQP-1 (Abcam, Cambridge, MA, USA) at a 1:500 dilution at $4^{\circ} \mathrm{C}$ in the blocking buffer. Furthermore, these membranes were washed with $0.1 \%$ Tween 20 and then treated with goat anti-rabbit IgG conjugated to alkaline phosphatase (1:2000) for 1 hour at $37^{\circ} \mathrm{C}$. The filters were stripped and reprobed for GAPDH normalization. The controls for 
nonspecific binding lacked primary antibodies. A film scanner (Image Master VDS; Amersham Biosciences, Inc., Piscataway, NJ) was used to scan the films.

Statistical Analysis

The statistical analyses were performed using version 17.0 SPSS software (SPSS, Inc., Chicago, IL, USA). All data are reported as the mean \pm standard deviation (SD) with a $95 \%$ confidence interval. Paired sample t-tests were used to compare the biometric data, which had a normal distribution, $₫$ between the experimental and normal control eyes, while data without a normal distribution were assessed by the Mann-Whitney U test. One-way analyses of variance (ANOVA) with a Bonferroni correction was performed for comparisons among the different groups. A $p<0.05$ was considered significant.

\section{Results}

\section{Refractive error and $\mathrm{AL}$}

At the beginning of the deprivation, there were no significant differences in refraction or AL between the experimental eyes and the normal control eyes $(p>0.05)$. After 21 days of form deprivation, the monocularly deprived eyes of the guinea pigs had developed significant degrees of myopia relative to the normal control eyes in terms of refractive error $(-0.86 \pm-0.53 \mathrm{D}$ vs $2.78 \pm 0.53 \mathrm{D}, p<0.001)$ and $\mathrm{AL}(8.25 \pm 0.11$ $\mathrm{mm}$ vs $7.92 \pm 0.11 \mathrm{~mm}, p<0.001)$.

In the REC and REC-DM groups, the changes in the refractive error (Fig. 1A) or AL (Fig. 1B) of the treated eyes regressed gradually after the deprivation was removed, and significant differences from the NC group were found in the REC $3 d$ and REC-DM $3 d$ groups (all $p<0.05$ ) but not in the REC 7d, REC 10d, RECDM $7 d$ and REC-DM 10d groups (all $p>0.05$ ). Furthermore, there were no significant differences between the REC group and the REC-DM group at the same time point (all $p>0.05$ ).

In the REC-AN groups, the changes in refractive error or AL regressed, and no significant differences were found in a comparison with the NC group at three time points (all $p>0.05$ ). In the REC-SP groups, there were significant differences in the refractive error only between the $3 \mathrm{~d}$ and $7 \mathrm{~d}$ groups and the NC group (both $p<0.05$ ), but for AL, significant differences were only found in the REC-SP $3 \mathrm{~d}$ group compared with the NC group ( $p=0.001)$. At 3 days after removal of the deprivation, the recovery of the REC-AN group was faster than that of the REC-SP, REC-DM, and REC groups in terms of AL and refractive error (all $p<0.05$ ), but the recovery of the REC-SP group was slower than that of the REC-AN, REC-DM, and REC groups in terms of $\mathrm{AL}$ and refractive error (all $p<0.05$ ); at 7 days, only the REC-SP group was significantly different from the REC-AN, REC-DM, and REC groups in refractive error (all $p<0.05$ ). At 10 days, there were no significant differences among the four groups (all $p>0.05$ ) (Fig. 1A, 1B).

Measurement of choroidal thickness

The CT in the eyes 21 days after deprivation $(62.6 \pm 6.9 \mu \mathrm{m})$ was significantly decreased compared with the CT in the eyes of the NC 21 group $(78.3 \pm 6.5 \mu \mathrm{m}, p<0.001)$. In the REC and REC-DM groups, a 
significant difference in CT was found between the NC group and the REC 3d or REC-DM $3 \mathrm{~d}$ groups ( $p=0.025, p=0.018$ ). Three days after the removal of the deprivation, the CT value in the REC-AN group was significantly higher than that in the other four groups (REC group, REC-SP group, REC-DM group and NC group) (all $p<0.05$ ) at the same time point, while the CT value in the REC-SP group was significantly lower than that in the other four groups (REC group, REC-AN group, REC-DM group and NC group) (all $p<0.05)$. At 7 days after the removal of the deprivation, there were no significant differences between the REC-AN 7d/REC 7d/REC-DM 7d groups and the NC 7d group in CT (all $p>0.05$ ), but significant differences were also found in the REC-SP 7d group compared with the NC $7 d$ group $(p=0.027)$. All experimental groups showed no significant difference from the normal control group at 10 days (all $p>$ 0.05) (Fig. 2A, 2B, 2C, 2D).

p-JNK1 and AQP-1 protein measurement

As shown in Fig. 3A, the protein expression of p-JNK1 and AQP-1 in the FD 21d group was significantly lower than that in the NC $21 \mathrm{~d}$ group $(0.27+0.06$ vs $0.63+0.14, p<0.001$ and $0.13+0.04$ vs $0.35+0.08$, $p<0.001)$. In the REC and REC-DM groups, there were significant differences in the p-JNK1 or AQP-1 protein expression in the REC3d, REC-DM $3 d$ and NC groups (all $p<0.05$ ), while no significant differences were found in the REC 7d, REC10d, REC-DM7d, and REC-DM10d groups compared with the NC group (all $p>0.05)$.

After 3 days of intravitreal injection of anisomycin, the p-JNK1 protein expression in the REC-AN 3d group was significantly increased compared with that of the other four groups (REC group, REC-SP group, RECDM group and NC group) (all $p<0.05$ ). We also found that the protein expression of AQP1 increased significantly compared with that of the other four groups (all $p<0.05$ ). There were no significant differences in the $\mathrm{p}-\mathrm{JNK} 1$ and AQP1 protein expression in the REC-AN7d and 10d groups compared with the NC group (all $p>0.05$ ).

In the REC-SP group, the $\mathrm{p}-\mathrm{JNK} 1$ and AQP-1 protein levels in the choroid were significantly reduced by SP600125 injection at 3 days after the removal of the deprivation compared with those of the REC-AN 3d group (both $p<0.001$ ), the REC $3 \mathrm{~d}$ group (both $p<0.05$ ), and the REC-DM 3d group (both $p<0.05$ ).

Significant differences were also found in the REC-SP $3 \mathrm{~d}$ and $7 \mathrm{~d}$ groups compared with the NC group (all $p<0.05$ ) in the protein expression of $p-J N K 1$ or AQP 1 , but there was no significant difference between the NC $10 \mathrm{~d}$ group and the REC-SP 10 group (both $p>0.05$ ).

\section{Discussion}

In this study, the p-JNK1 and AQP-1 protein expression in the FD 21d group appeared to be decreased in the choroid compared with that in the NC $21 \mathrm{~d}$ group; then, the expression increased after the face mask was removed and returned to normal at 7 days after recovery. We also found an association between changes in JNK1 signaling and recovery from myopia in the present study. Specifically, elevation of the pJNK1 levels by the JNK1 stimulator anisomycin accelerated the axial shortening and recovery from myopia. Consistent with this finding, SP600125, a JNK1 inhibitor, decreased the p-JNK1 levels and 
inhibited the recovery from myopia. These results indicate that JNK1 may play a role in the recovery from myopia and may participate in the rehabilitation mechanism of myopia. These data are consistent with the findings of Brand $\mathrm{C}$ et al. [11].

We found that the inhibition of the JNK1 pathway decreased the expression of AQP-1 in the choroid, while the activation of the JNK1 pathway promoted the activation of AQP-1. In previous studies on AQP1 activation, Umenishi $F$ et al. also found that rat bone marrow cells can increase AQP-1 expression by activating JNK, while inhibition of JNK could significantly reduce AQP-1 expression [12], indicating that the JNK pathway can also affect AQP1 activation.

The mechanism for the regulation of AQP-1 expression is as follows: first, the AQP1 transcript is upregulated by a hypertonicity response element (HRE) in the promoter region of the AQP1 gene, which can be activated by hypertonicity through three mitogen-activated protein kinases (MAPK) (ERK, p38 kinase, and JNK) signaling pathways. Therefore, the osmotic regulation of AQP1 may be mediated by JNK pathways, which are induced by hypertonicity [12]. Chaoyang Zhang et al. found that erythropoietin maintained the integrity of the outer blood-retinal barrier and retinal edema by downregulating HIF-1a and JNK signals, thus upregulating the expression of ZO-1 and occludin in the RPE cells of early diabetic rats [15]. This finding indicates that JNK may also affect the permeability of blood vessels, thus changing the osmotic pressure inside and outside the vessels. Second, AQP1, -4 , and -9 have an adaptor primers 1 (AP1) element in their promoter region, allowing JNK-dependent transcriptional regulation [16]. Furthermore, AQP1 may lead to increased vascular permeability and interstitial fluid effusion, thus causing choroid thickening [17]. Third, JNK can induce the activation of p-c-Jun and cleaved caspase-3, which is related to the activation of the aquaporin system. Hiroshi Yatsushige et al. found that activated JNK can activate the AQP of the neurovascular unit in early brain injury in this way [18]. However, Lihua Liu et al. found that the ERK/JNK/p38 pathways are involved in the downregulation of AQP1 via activation of phosphorylated components, thus regulating the expression of AQP-1 in MeT-5A cells [19]. The main reasons for the discrepancy with our findings is that stimulation is different. In their study, the cells were treated with peptidoglycan and lipopolysaccharide in vitro. Further studies of the activation of MAPKs by upstream kinases (including MEK or MKKs) and downstream transcription factors (including NF-KB and ATF-2) are needed to elucidate the detailed regulatory mechanism.

In our experiment, we found that the CT gradually recovered after removal of the deprivation. Moreover, compared with that of the control group, the recovery with the JNK1 agonist was faster, while the recovery with the inhibitor was slower. In our previous research, we also found a significant positive correlation between AQP-1 and CT in FD and the recovery period, indicating that the regulation of AQP-1 expression is related to changes in the $\mathrm{CT}$ [3]. The expansion of the choroid in the recovery period reflects the expansion of the choroidal lacunae, while the thinning of the choroid during form deprivation reflects the reduction of the choroidal lacunae. The changes in the thickness of the lacuna during the expansion and thinning of the choroid indicate that CT can be regulated by fluid exchange between the lymphatic and vascular components. Therefore, the upregulation of AQP-1 expression may be detrimental to the removal of water from choroidal capillaries to choroidal lacunae. Sen Zhang et al. also found that choroidal blood 
perfusion increased during the recovery from FD myopia, which could result in increased water levels in the blood vessels [20]. Therefore, we speculate that JNK may regulate CT by regulating the expression of AQP1 in the choroid.

There are several limitations in our study. First, this study only measured the protein expression of $\mathrm{p}$ JNK1; p38 and other cytokines were not examined. Many signal factors can affect AQP-1. Cytokines play an important role in the control of CT and AQP-1 in the occurrence of and recovery from FD myopia. Therefore, future research should explore the roles of other cytokines in the pathogenesis of myopia. Second, in this experiment, we did not observe toxicity of the intravitreal injections of drugs or toxicity and side effects on the retina and choroid. Yasunari Munemasa et al. found that a JNK inhibitor was effective not only in ameliorating N-methyl-D-aspartate-induced apoptotic cell loss in the retinal ganglion cell layer but also in protecting the inner retina when $0.2 \mathrm{nmol}$ SP600125 was injected into the vitreous cavity of rats [21]. Claudia B.L et al found $0.1 \mu \mathrm{g} / \mathrm{ml}$ of anisomycin promoted a strong activation of p38 without inducing cell death in the retina[22]. In our experiment, we choose $0.2 \mathrm{nmol}$ of anisomycin and SP600125 for intravitreal injections. Third, FD does not represent the actual cause of myopia. Previous studies have reported that environmental factors and genetics influence myopia in the real world [23], but FD can be studied as an experimental method. Fourth, since the software for automatic CT measurement is not available, CT measurement is performed manually [24]. In this study, when the measurement difference was more than $5 \%$, two blinded investigators made a consensus judgment. Fifth, we did not observe changes in the contralateral control eyes in our study because the injection may affect the contralateral eye and cause errors. In future studies, we will observe the contralateral eye.

\section{Conclusions}

In summary, we found that the levels of p-JNK1 and AQP-1 in the choroid and CT were higher than those in the normal control eyes during the recovery from myopia and returned to normal at 7 days after removal of the deprivation. More importantly, activation of JNK1 by anisomycin upregulated the AQP-1 protein level, which could accelerate the recovery from myopia and rapidly increase $\mathrm{CT}$. In addition, inhibition of JNK1 activity by SP 60025 decreased the level of AQP-1, and the thickening of CT was retarded, thus attenuating the recovery from myopia. These findings indicate that the JNK1 pathway plays a critical role in controlling the recovery from myopia by regulating CT and the AQP-1 content in the choroid. Further research on the role of changes in JNK1 signal transduction during the recovery from myopia may identify potential drug targets for treatment of myopia.

\section{Declarations}

\section{Ethics approval and consent to participate}

This study was approved by the Ethics Committee of the Tian Jin Eye Hospital, and was conducted in accordance with the Association for Research in Vision and Ophthalmology Statement for the Use of Animals in Ophthalmology and Vision Research and the Declaration of Helsinki. 


\section{Consent to publish}

Not applicable.

\section{Availability of data and materials}

The datasets used and/or analyzed during the current study are available from the corresponding author on reasonable request at any time.

\section{Competing interests}

The authors declare that they have no competing interests.

\section{Funding}

The authors are thankful for the financial support of grant 17JCYBJC26900 from the Tian Jin Science Foundation of China and 2019110 from the Tian Jin Health Commission of China.

\section{Authors' contributions}

WC and YZ designed the study, analyzed the data and wrote the manuscript. WC, ZW L, QM W performed the animal study. YW and $Y Z$ revised and made suggestions to the manuscript. All authors read and approved the final manuscript.

\section{Acknowledgments}

The authors wish to acknowledge the support of Peng Hao MD, Bin Wu MD, Yu Chuan Wang MD, Jing Li MD, Ruifang Han MD, Ze Yu Liang MD®Ming Ying MD at the Tianjin Eye Hospital, Nankai University Affiliated Eye Hospital, Clinical College of Ophthalmology.

\section{Conflict of Interest}

no conflicting relationship exists for any author.

\section{Abbreviations}

AL: axial length; AP1: adaptor primers 1; AQP-1: Aquaporin-1; CT: choroidal thickness; FD: form deprivation; JNK1:C-Jun N-terminal kinase-1; MAPK: mitogen-activated protein kinases; NC: normal control; OCT: optical coherence tomography; REC: recovery; REC-AN: REC plus anisomycin; REC-DM: REC plus DMSO; REC-SP: REC plus SP600125; RPE: retinal pigment epithelium; SD: standard deviation;

\section{References}

1. Cooper J, Tkatchenko AV. A Review of Current Concepts of the Etiology and Treatment of Myopia. Eye Contact Lens. 2018;44:231-247. 
2. Zhou X, Ye J, Willcox MD, et al. Changes in protein profiles of guinea pig sclera during development of form deprivation myopia and recovery. Mol Vis. 2010;16:2163-2174.

3. Chen W, Zhang H, Zhang Y, Wang Q, Wang Y, Li ZW. Relationship between Aquaporin-1 Protein Expression and Choroidal Thickness during the Recovery of Form-deprivation Myopia in Guinea Pigs. Curr Eye Res. 2020;45:705-712.

4. Nickla DL, Wallman J. The multifunctional choroid.Prog Retin Eye Res. 2010,29:144-68.

5. Okamoto M, Matsuura T, Ogata N. Choroidal thickness and choroidal blood flow after intravitreal bevacizumab injection in eyes with central serous chorioretinopathy. Ophthalmic Surg Lasers Imaging Retina. 2015;46:25-32.

6. Junghans BM, Crewther SG, Liang H, Crewther DP. A role for choroidal lymphatics during recovery from form deprivation myopia? Optometry Vis Sci. 1999;76:796-803.

7. Woodman-Pieterse EC, Read SA, Collins MJ, Alonso-Caneiro D. Regional Changes in Choroidal Thickness Associated With Accommodation.Invest Ophthalmol Vis Sci. 2015;56:6414-6422.

8. Nickla DL, Wildsoet C, Wallman J. Compensation for spectacle lenses involves changes in proteoglycan synthesis in both the sclera and choroid. Curr Eye Res. 1997;16:320-26.

9. Hotamisligil GS, Davis RJ. Cell Signaling and Stress Responses.Cold Spring Harb Perspect Biol. 2016;8:a006072.

10. Brand C, Schaeffel F, Feldkaemper MP.A microarray analysis of retinal transcripts that are controlled by image contrast in mice. Mol Vis. 2007,13:920-32

11. Umenishi F, Schrier RW.Hypertonicity-induced aquaporin-1 (AQP1) expression is mediated by the activation of MAPK pathways and hypertonicity-responsive element in the AQP1 gene. J Biol Chem. 2003,278:15765-70.

12. Chen W, Song H, Xie S, Han Q, Tang X, Chu Y. Correlation of macular choroidal thickness with concentrations of aqueous vascular endothelial growth factor in high myopia.

Curr Eye Res. 2015;40:307-13.

13. Papastergiou GI,Schmid GF,Riva CE,Mendel MJ,Stone RA,Laties AM.

Ocular axial length and choroidal thickness in newly hatched chicks and one-yearold chickens fluctuate in a diurnal pattern that is influenced by visual experience and intraocular pressure changes. Exp Eye Res.1998;66:195-205.

14. Zhang $\mathrm{C}$, Xie H, Yang Q, et al. Erythropoietin protects outer blood-retinal barrier in experimental diabetic retinopathy by up-regulating ZO-1 and occludin. Clin Exp Ophthalmol. 2019;47:1182-1197.

15. Tsukaguchi H, Weremowicz S, Morton CC, Hediger MA. Functional and molecular characterization of the human neutral solute channel aquaporin-9.Am J Physiol. 1999;277:F685-F696.

16. Mobasheri A, Airley R, Hewitt SM, Marples D. Heterogeneous expression of the aquaporin 1 (AQP1) water channel in tumors of the prostate, breast, ovary, colon and lung: a study using high density multiple human tumor tissue microarrays. Int J Oncol. 2005;26:1149-1158. 
17. Yatsushige H, Ostrowski RP, Tsubokawa T, Colohan A, Zhang JH. Role of c-Jun N-terminal kinase in early brain injury after subarachnoid hemorrhage.J Neurosci Res. 2007;85:1436-1448.

18. Liu L, Xie C. Effects of downregulation of aquaporin 1 by peptidoglycan and lipopolysaccharide via MAPK pathways in MeT-5A cells. Lung. 2011;189:331-340.

19. Zhang S, Zhang G, Zhou X, et al. Changes in Choroidal Thickness and Choroidal Blood Perfusion in Guinea Pig Myopia.Invest Ophthalmol Vis Sci. 2019;60:3074-3083.

20. Munemasa Y, Ohtani-Kaneko R, Kitaoka Y, et al. Contribution of mitogen-activated protein kinases to NMDA-induced neurotoxicity in the rat retina.Brain Res. 2005;1044:227-240.

21. Campos CB, Bédard PA, Linden R. Requirement of p38 stress-activated MAP kinase for cell death in the developing retina depends on the stage of cell differentiation. Neurochem Int. 2006;49(5):494499 .

22. Myrowitz EH. Juvenile myopia progression, risk factors and interventions. Saudi J Ophthalmol. 2012;26:293-7.

23. Chen W, Li L, Zhang H, Li Y, Chen X, Zhang Y. Macular choroidal thickness in highly myopic women during pregnancy and postpartum: a longitudinal study. BMC Pregnancy Childbirth. 2018;18:220.

\section{Table}

Table 1. Experimental animal group classification ( $\mathrm{n}=8$ in each group)

\begin{tabular}{|c|c|c|c|c|c|}
\hline \multirow[t]{2}{*}{ Groups } & \multicolumn{3}{|c|}{$\begin{array}{l}\text { Time after removal of deprivation (or the } \\
\text { corresponding time in the NC group) }\end{array}$} & \multirow{2}{*}{$\begin{array}{c}\begin{array}{c}\text { Time of } \\
\text { deprivation }\end{array} \\
21 \text { days } \\
\end{array}$} & \multirow[t]{2}{*}{$\begin{array}{c}\text { Intravitreal } \\
\text { injection }\end{array}$} \\
\hline & 3 days & 7 days & 10 days & & \\
\hline $\mathrm{REC}$ & REC 3d & REC 7d & REC 10d & & \\
\hline $\begin{array}{l}\text { REC- } \\
\text { SP }\end{array}$ & REC-SP 3d & REC-SP 7d & REC-SP 10d & & SP600125 \\
\hline $\begin{array}{l}\text { REC- } \\
\text { AN }\end{array}$ & REC-AN 3d & REC-AN 7d & REC-AN 10d & & anisomycin \\
\hline $\begin{array}{l}\text { REC- } \\
\text { DM }\end{array}$ & REC-DM 3d & REC-DM 7d & REC-DM 10d & & DMSO \\
\hline FD & & & & FD 21d & \\
\hline$\overline{\mathrm{NC}}$ & $\mathrm{NC} 3 \mathrm{~d}$ & $\mathrm{NC} 7 \mathrm{~d}$ & NC 10d & NC 21d & \\
\hline
\end{tabular}

REC: recovery; REC-SP: REC plus SP600125; REC-AN: REC plus anisomycin; REC-DM: REC plus DMSO; NC: normal control. NC 3d group mean 45 days old guinea pigs in normal control group, NC 7d group mean 49 days old guinea pigs in normal control group, NC 10d group mean 52 days old guinea pigs in normal control group

\section{Figures}




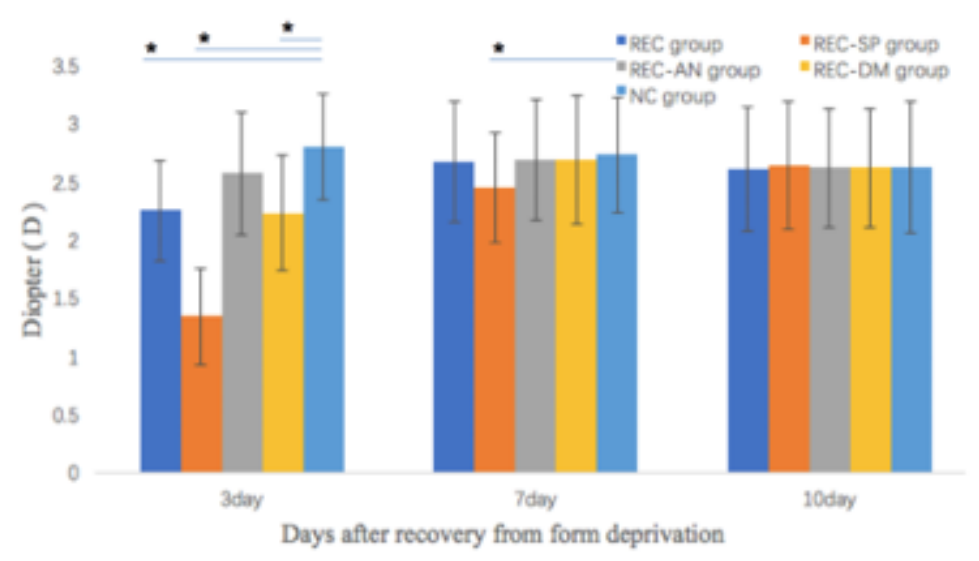

Fig. 1A

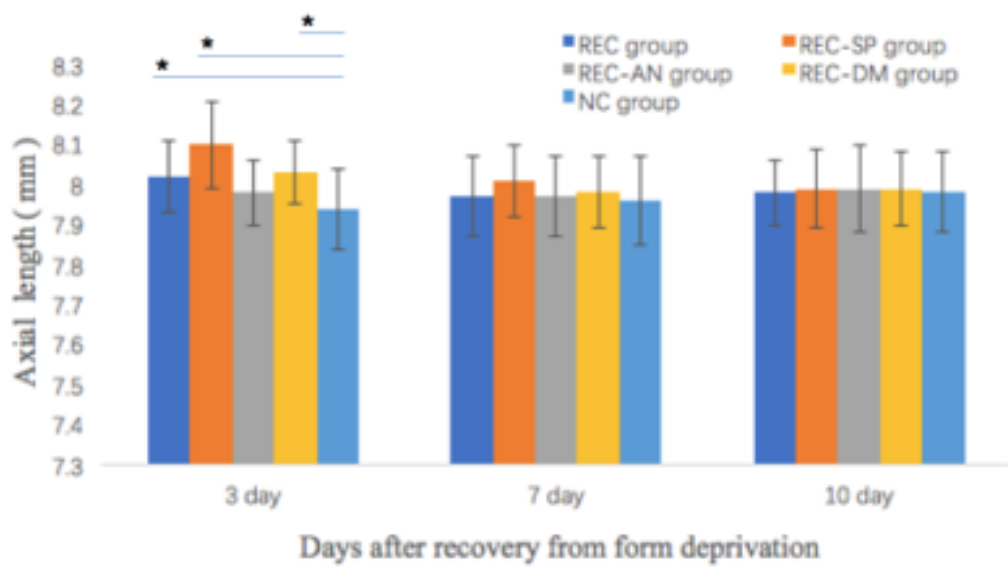

Fig. 1B

\section{Figure 1}

Mean refractive error $(A)$ and axial length $(B)$ at different time points after removal of the deprivation in the recovery group (REC group), the REC plus SP600125 group (REC-SP group), the REC plus anisomycin group (REC-AN group), the REC plus DMSO group (REC-DM group) and the normal control group (NC group). $* p<0.05$. 


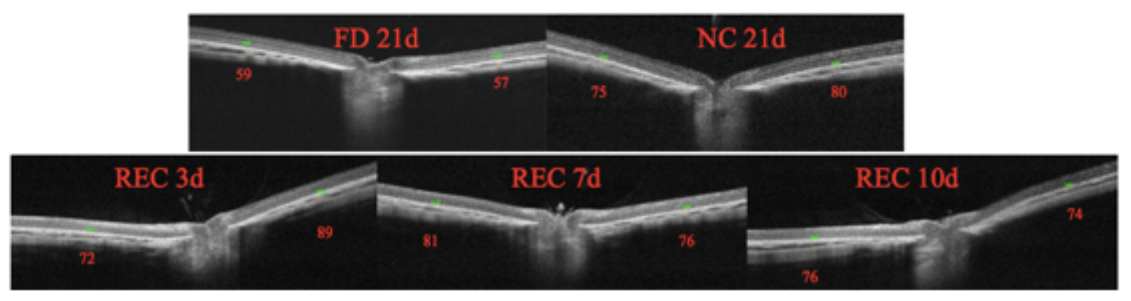

Fig. 2A

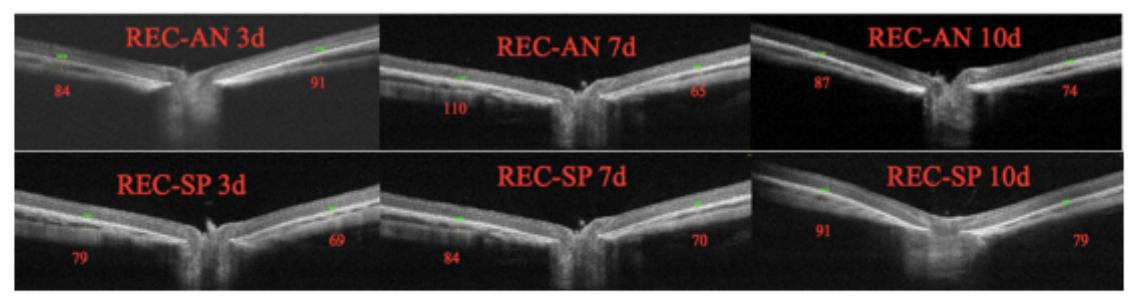

Fig. 2B

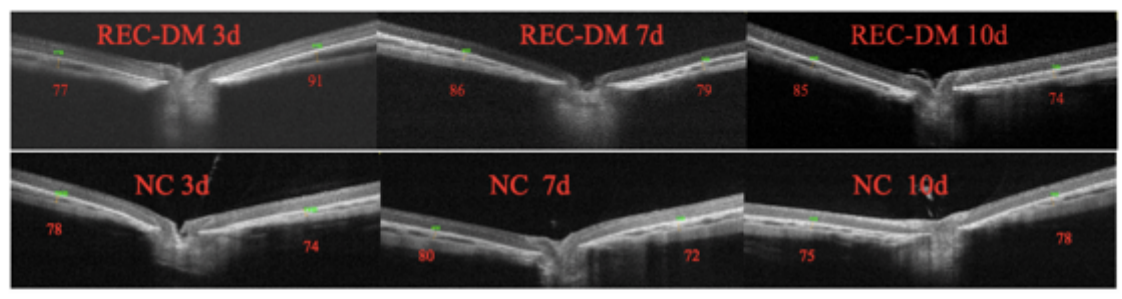

Fig. 2C

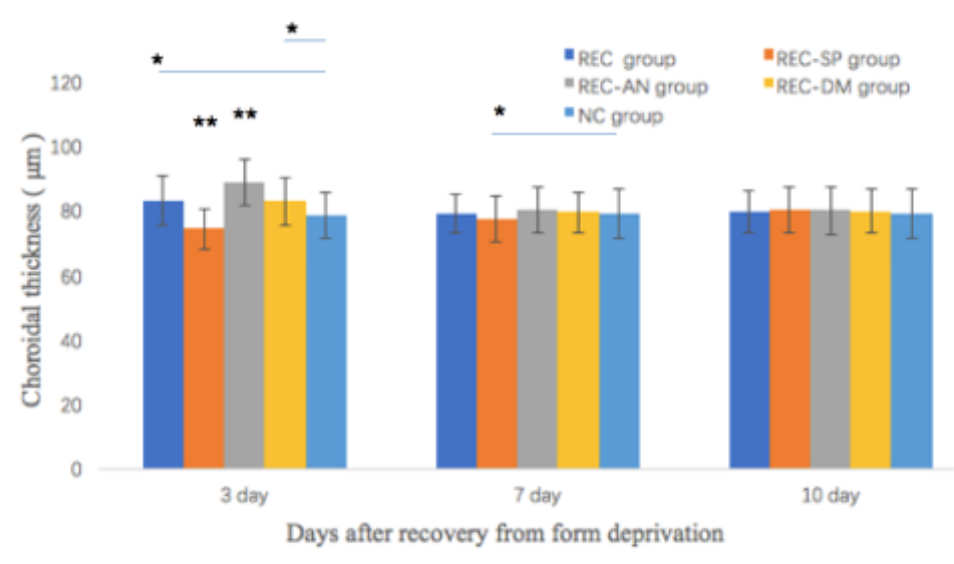

Fig. 2D

\section{Figure 2}

(A, B , C) The OCT horizontal cross-sections in the form deprivation 21 day (FD 21d) group, different time points after removal of the deprivation in the recovery (REC, REC-SP, REC-AN, REC-DM) group and different time points in the normal control (NC) group. The choroidal thickness (CT) was defined as the vertical from the outer surface of the hyperreflective line ascribed to the retinal pigment epithelial to the hyperreflective line of the inner sclera border (line). (D) Mean CT at different time points after the removal 
of the deprivation in the REC group, the REC-SP group, the REC-AN group, the REC-DM group and the NC group. The results are the geometric mean and the $95 \% \mathrm{Cl} . * \mathrm{p}<0.05$; $* *$ : compared with the other groups at the same time point, $p<0.05$.
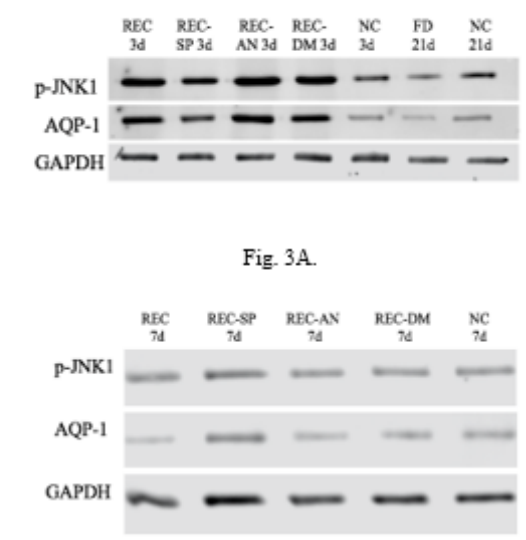

Fig. 3B

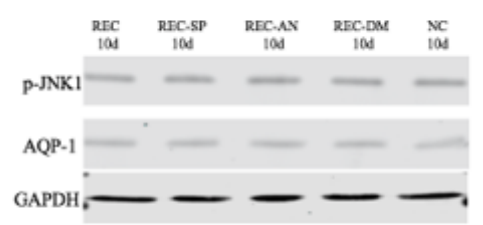

Fig. 3C

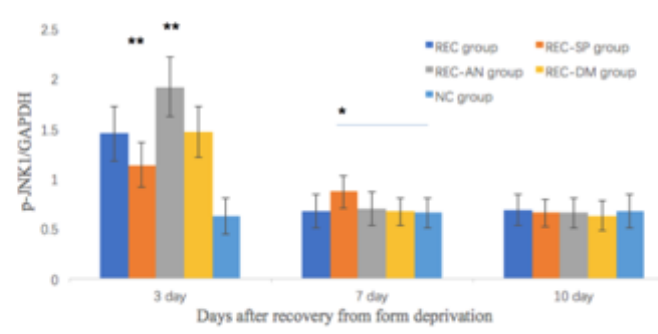

Fig. 3D

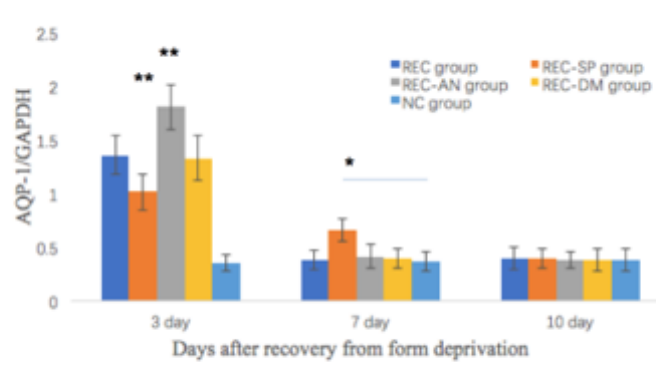

Fig. 3E

\section{Figure 3}

(A, B, C) The p-JNK 1 and AQP-1 protein levels were measured by Western blots in the form deprivation 21 days (FD 21d) group at different time points after removal of the deprivation in the recovery (REC, REC-SP, 
REC-AN, REC-DM) group and at different time points in the normal control (NC) group. (D, E) Mean p-JNK 1 and AQP-1 protein levels at different time points after removal of the deprivation in the REC group, the REC-SP group, the REC-AN group, the REC-DM group and the NC group. The results are the geometric mean and $95 \% \mathrm{Cl} . * \mathrm{p}<0.05$; $* *$ : compared with the other groups at the same time point, $\mathrm{p}<0.05$. GAPDH $\mathbb{}$ glyceraldehyde-3phosphate dehydrogenase .

\section{Supplementary Files}

This is a list of supplementary files associated with this preprint. Click to download.

- renamed6654e.docx

- NC3RsARRIVEGuidelinesChecklistfillable.pdf 